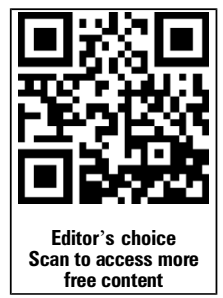

- Additional material is published online only. To view please visit the journal online (http://dx.doi.org/10.1136/ flgastro-2015-100613).

For numbered affiliations see end of article.

\section{Correspondence to} Dr Joseph Low, Marie Curie Palliative Care Research Department, Division of Psychiatry, UCL, 6th Floor Maple House, 149 Tottenham Court Road, London W1T 7NF, UK; joseph.low@ucl.ac.uk

Received 11 May 2015 Revised 17 June 2015 Accepted 2 July 2015 Published Online First 24 July 2015
CrossMark

To cite: Low J, Vickerstaff $\mathrm{V}$, Davis $\mathrm{S}$, et al. Frontline

Gastroenterology 2016;7:4-9.

\title{
Palliative care for cirrhosis: a UK survey of health professionals' perceptions, current practice and future needs
}

\author{
Joseph Low, ${ }^{1}$ Victoria Vickerstaff, ${ }^{1}$ Sarah Davis, ${ }^{1}$ Julia Bichard, ${ }^{2}$ \\ Lynda Greenslade, ${ }^{3}$ Katherine Hopkins, ${ }^{2}$ Aileen Marshall, ${ }^{3}$ \\ Douglas Thorburn, ${ }^{3,4}$ Louise Jones ${ }^{1}$
}

\begin{abstract}
Objective To determine the knowledge and practice patterns of a UK cohort of relevant healthcare professionals (HCPs) about delivering palliative care in cirrhosis, and to inform priorities for future research.
\end{abstract}

Design An on-line questionnaire survey with closed and open responses.

Setting HCPs identified from the mailing list of special interest groups in hepatology and gastroenterology (liver), general practice and specialist palliative care (SPC) across the UK.

Results Of the 6181 potential contacts identified, 517 HCPs responded. Most believed a role exists for SPC in caring for people with cirrhosis, but many SPC HCPs felt ill prepared to provide good care to those facing death. Further training was needed in managing liver-related symptoms, symptom control and end of life issues. All HCP groups wished to increase community provision of palliative care support, but many general practitioners felt unable to manage advanced cirrhosis in the community. There were differences in the optimal trigger for SPC referral with liver HCPs less likely to refer at symptom deterioration. Prognostication, symptom management and service configuration were key areas identified for future research.

Conclusions All who responded acknowledged the role of SPC in caring for those dying with cirrhosis and need for further training to improve confidence and enable joint working between SPC, general practice and liver teams. Low response rates make it difficult to generalise these findings, which require further validation.

\section{INTRODUCTION}

Liver disease is the third largest cause of premature death in the $\mathrm{UK}^{1}$ and accounts for $2 \%$ of deaths in England. ${ }^{2}$ The increases in alcohol consumption, viral hepatitis and obesity in the UK suggest that liver disease is a growing public health problem. ${ }^{3}$ People with end stage liver disease (cirrhosis) have supportive and palliative care needs. ${ }^{4-7}$ The recent Lancet Commission report on improving liver care acknowledges the importance of primary care and hospital services in providing high quality care for people with cirrhosis. ${ }^{1}$ Nevertheless it skims over the care issues arising for those who are dying of liver disease. At present, this care is poor, ${ }^{8}$ most deaths from cirrhosis occur in hospital ${ }^{2}$ and more work is needed to understand how end of life (EoL) care for people with cirrhosis can be managed well.

The Royal Free Hospital London (tertiary centre) liver team and the Marie Curie Palliative Care Research Department, University College London held a national meeting of health professionals interested in these aspects of liver care in November 2013. The group recommended a national survey of primary, secondary and tertiary care providers to explore current attitudes and practices, and to identify training needs, ideas for service improvement and research priorities. Here we report the findings of this survey.

\section{METHODS}

We developed an online survey (see online supplementary appendix ii) to explore (A) referrals to specialist palliative care (SPC) - numbers, reasons, timing; (B) health professionals' training needs; 
(C) ideas for improved EoL care in cirrhosis; (d) areas for future research.

The link to the survey and an invitational email were sent to (1) those working in liver teams; (2) those working in SPC; (3) general practitioners (GPs) with an interest in gastroenterology. To identify potential participants, we gained the cooperation of key professional organisations who contacted their members with two reminders at 3-week intervals (see online supplementary appendix ii):

1. Liver teams: (A) the British Society for Gastroenterology (BSG) (liver section); (B) the British Association for the Study of the Liver (BASL) (doctors and nurses); (C) the British Liver Trust Nurses Forum; (D) Royal College of Nurses (gastroenterology forum) and (E) the London Liver group.

2. SPC teams: (A) Association of Palliative Medicine of Great Britain and Ireland; (B) the National Nurse Consultant Group (palliative care); (C) Clinical and nursing leads in SPC units ('Help the Hospice' database).

3. General Practitioners: the Primary Care Society for Gastroenterology.

We used descriptive statistics to explore data on responders' demographics, referrals to SPC, training needs in caring for people with cirrhosis and key areas for research. Open-ended questions were analysed using frequency content analysis after which we triangulated quantitative data and open responses. Statistical analyses were conducted using Stata V.13.0 ${ }^{9}$

\section{RESULTS}

A maximum of 6181 contacts received the online survey and we received data from 514 respondents: 195 liver professionals, 273 SPC and 46 general practice professionals (response rates $4 \%, 23 \%$ and $10 \%$, respectively; overall response rate $8 \%$ ). A breakdown of responders by specialty and discipline (see online supplementary appendix iii) showed that most were from a medical background (306/514) and worked in SPC (273/514). Analysis by National Health Service (NHS) Region (see online supplementary appendix iv) showed responses across the UK, with most from the north of England.

\section{Referrals to SPC}

Nearly all SPC professionals (97\%; 262/269) had accepted referrals from liver teams for patients with cirrhosis near the EoL; most $(86 \% ; 232 / 269)$ had been referred at least one patient during the last year. However, patients with cirrhosis made up a median 1\% of SPC workload (IQR: 1-2, range: $0-20$ ).

Numbers of referrals reported by liver professionals (table 1) to SPC were low; most referred fewer than 11 patients a year (table 1) and these were more likely to be inpatients than outpatients (table 1). Referrals from GPs were equally low, with only $19 \%(44 / 228)$ of SPC professionals receiving referrals for patients with cirrhosis, and no more than five patients per year.
Reasons for referrals to SPC, from liver professionals and GPs were symptom control, terminal care and the need for hospice admission (table 2). Most liver and SPC professionals identified recognition of the EoL as the key trigger for SPC referral in cirrhosis. SPC professionals also considered onset of deterioration in symptoms and recurrent hospital admissions as appropriate triggers for referral to their service (table 2).

\section{EoL care for cirrhosis in primary care}

Over half of GPs $(55 \% ; 22 / 40)$ felt their patients could be managed in the community, but only $24 \%$ $(10 / 41)$ had actually received a referral from liver centres. Confidence in managing patients in the community was attributed to good support from community nursing or local SPC teams. Barriers were the perceived unpredictable nature of cirrhosis and patients' social circumstances (especially those actively drinking).

\section{Skills levels and training needs}

Although most SPC professionals $(60 \% ; 147 / 246)$ and GPs $(54 \% ; 22 / 41)$ were confident in their skills and knowledge to manage people with cirrhosis, all recognised the need for further training on issues specific to liver disease; liver professionals and GPs identified symptom control and training in EoL issues as the two key topics to address (table 3 ).

Open response data showed that liver professionals need improved awareness of supportive care including symptom control at EoL, advanced communication skills in discussing prognosis with patients and families, and help to shift the emphasis of care from an interventionist to a more supportive role; liver personnel and GPs need improved knowledge on how SPC could support them to care for people with advanced cirrhosis and the processes of accessing community SPC.

Liver professionals felt that primary care and SPC needed specific training in understanding the

Table 1 The number of patients referred to specialist palliative care (SPC) from liver professionals by type of care and diagnosis

\begin{tabular}{|c|c|c|c|c|c|c|}
\hline & \multicolumn{4}{|c|}{$\begin{array}{l}\text { Number referred by liver } \\
\text { professionals to SPC }\end{array}$} & \multirow{2}{*}{\multicolumn{2}{|c|}{$\begin{array}{l}\begin{array}{l}\text { Number of } \\
\text { referrals } \\
\text { received by SPC }\end{array} \\
\text { Cirrhosis }\end{array}$}} \\
\hline & \multicolumn{2}{|c|}{ Inpatient care } & \multicolumn{2}{|c|}{ Outpatient care } & & \\
\hline & $\mathrm{N}$ & Per cent & $\mathrm{N}$ & Per cent & $\mathrm{N}$ & Per cent \\
\hline None & 7 & 4 & 71 & 44 & 37 & 14 \\
\hline $1-5$ & 45 & 28 & 51 & 32 & 158 & 59 \\
\hline $6-10$ & 49 & 30 & 14 & 9 & 45 & 17 \\
\hline $11-20$ & 38 & 24 & 16 & 10 & 25 & 9 \\
\hline $21+$ & 22 & 14 & 9 & 6 & 4 & 1 \\
\hline Total & 161 & & 161 & & 269 & \\
\hline
\end{tabular}




\begin{tabular}{|c|c|c|c|c|c|c|}
\hline & \multicolumn{2}{|c|}{ Liver professionals } & \multicolumn{2}{|c|}{ General practice } & \multicolumn{2}{|c|}{$\begin{array}{l}\text { Specialist palliative } \\
\text { care }\end{array}$} \\
\hline & $\mathrm{N}$ & Per cent & $\mathrm{N}$ & Per cent & $\mathrm{N}$ & Per cent \\
\hline \multicolumn{7}{|l|}{ Reasons for referral to SPC by specialty } \\
\hline Symptom Control & 137 & 88 & 12 & 75 & & \\
\hline End of life issues & 138 & 89 & 12 & 75 & & \\
\hline Hospice/palliative care & 123 & 79 & 9 & 56 & & \\
\hline Social/family & 73 & 47 & 8 & 50 & & \\
\hline Bereavement support & 39 & 25 & 3 & 19 & & \\
\hline Ethical decision making & 11 & 7 & 2 & 13 & & \\
\hline Communicating difficult news & 7 & 5 & 1 & 6 & & \\
\hline Other & 3 & 2 & 2 & 13 & & \\
\hline Total & 155 & & 16 & & & \\
\hline \multicolumn{7}{|l|}{ Most appropriate times for referral to SPC by specialty } \\
\hline End-stage/end of life & 158 & 89 & & & 223 & 91 \\
\hline Deterioration in symptoms & 105 & 59 & & & 198 & 81 \\
\hline Recurrent hospital admissions & 111 & 63 & & & 180 & 74 \\
\hline $\begin{array}{l}\text { Consideration of referral to tertiary liver centre, for example, } \\
\text { for liver transplant assessment }\end{array}$ & 32 & 18 & & & 59 & 24 \\
\hline Initial diagnosis & 12 & 7 & & & 21 & 9 \\
\hline Other & 13 & 7 & & & 0 & 0 \\
\hline Total responses & 177 & & & & 245 & \\
\hline
\end{tabular}

SPC, specialist palliative care.

unpredictable trajectory of cirrhosis and the impact of impaired liver function on medication metabolism. SPC professionals recognised their training needs on liver specific issues such as the assessment and management of decompensated liver disease $(86 \% ; 198 /$ 231), pharmacology in cirrhosis $(79 \% ; 182 / 231)$, likely illness trajectory in cirrhosis $(68 \% ; 159 / 231)$, advances in the management of recurrent ascites
$(68 \% ; 158 / 231)$ and information on prognostication (68\%; 158/231).

Initiatives for improving EoL care in cirrhosis

Liver professionals suggested enhanced provision of palliative care whereas most SPC professionals prioritised improved liaison between secondary and primary care. GPs also favoured improved liaison (33/

Table 3 Comparison of training needs and initiatives to improve end of life care for patients with cirrhosis by specialty

\begin{tabular}{|c|c|c|c|c|c|c|}
\hline & \multicolumn{2}{|c|}{ Liver professionals } & \multicolumn{2}{|c|}{ General practice } & \multicolumn{2}{|c|}{$\begin{array}{l}\text { Specialist palliative } \\
\text { care }\end{array}$} \\
\hline & $\mathrm{N}$ & Per cent & $\mathrm{N}$ & Per cent & $\mathrm{N}$ & Per cent \\
\hline \multicolumn{7}{|c|}{ Comparison of training needs of liver and general practice professionals } \\
\hline Symptom control & 101 & 66 & 31 & 91 & & \\
\hline End of life issues & 101 & 66 & 18 & 53 & & \\
\hline Ethical decision making & 55 & 36 & 9 & 27 & & \\
\hline Bereavement counselling & 52 & 34 & 7 & 21 & & \\
\hline Social/family & 45 & 29 & 8 & 24 & & \\
\hline Communicating difficult news & 40 & 26 & 8 & 24 & & \\
\hline Other & 4 & 3 & 2 & 6 & & \\
\hline Total & 154 & & 34 & & & \\
\hline \multicolumn{7}{|c|}{ Initiatives that would improve end of life care for patients with cirrhosis by specialty } \\
\hline Improved liaison between secondary and primary care & 69 & 39 & & & 90 & 37 \\
\hline Improved provision of specialist palliative care & 86 & 48 & & & 64 & 26 \\
\hline More education opportunities in specialist liver units & 21 & 12 & & & 71 & 29 \\
\hline Other & 3 & 2 & & & 20 & 8 \\
\hline Total & 179 & & & & 245 & \\
\hline
\end{tabular}


$39 ; 85 \%$ ), more educational opportunities in specialist liver units $(26 / 39 ; 67 \%)$ and improved provision of SPC (18/39; 46\%).

Analysis of open responses from 137/195 liver professionals (88 doctors and 49 nurses), identified specific and more detailed improvements which could be made to the delivery of EoL care, in reconfiguring services and improving clinicians' skill base:

i. Earlier SPC involvement: Significant time points in the patient journey, such as assessment for liver transplantation, repeated hospital admissions or onset of need for regular paracentesis could prompt SPC referral. Understanding of appropriate triggers and markers of deterioration may support health professionals to recognise when symptomatic patients would benefit from SPC which can be delivered in parallel with more aggressive treatments.

ii. Better community provision of liver services: Better liaison between liver teams, primary care and SPC could enable patients to receive community nursing and paracentesis, enhanced by hospice outpatient services to provide support and symptom control as required, which may reduce emergency admissions to hospital.

iii. Development of clearer care pathways: Understanding of care pathways would guide liver professionals to identify which patients with cirrhosis had reached the EoL phase and what to do next. An EoL pathway would provide a framework to consider withdrawal of medication and opportunities for patients and those close to them to access counselling and support.

iv. Ways of improving communication skills: Liver professionals recognised that patients and family members had little understanding about their disease or its severity. At the same time, they themselves lacked confidence in having honest discussions about these topics. Sharing of information about the disease, its likely trajectory and symptoms might allow more open discussions about future care, and choices about place of care, interventions and services available.

\section{Key priorities for future research}

Open responses on research priorities were received from 221 participants. Table 4 highlights the key findings. (1) Disease specific information-all groups highlighted the need to understand: symptom control (in particular how best to manage recurrent ascites, pruritus and encephalopathy, and opioid pharmacology when liver function is compromised), and prognostication (in particular identifying the earliest point when deterioration is irreversible); (2) Service models -SPC and liver professionals prioritised exploring whether palliative care adds value (economical or quality of life), the best way of managing patients in the community to avoid hospital admissions, and the evaluation of an early palliative care intervention versus usual care; understanding would be enhanced by exploring the experience of patient and their family members in (A) their understanding of prognosis and their own wishes about future preference of care (B) what specific needs they have as a result of the illness.

\section{DISCUSSION}

This is the first UK-based national survey on the perspectives of health professionals responsible for delivering care to people with cirrhosis who are facing death.

Referrals from liver teams to SPC are relatively uncommon and usually made late in the illness when death is very close. Understanding of the role of SPC was poor. All health professionals, irrespective of specialty, recognised that they required further training to improve their skills in caring for people with advanced cirrhosis. For SPC, this training centred on increasing awareness of specific issues involved in managing patients with liver disease, such as the implications of impaired liver function on symptom control and medication use. For liver professionals, future training should support them to identify

Table 4 Research themes identified by discipline

\begin{tabular}{|c|c|c|c|c|}
\hline & SPC $(n=114)$ & Liver professionals ( $n=93$ ) & General practice $(n=14)$ & Total $(n=221)$ \\
\hline \multicolumn{5}{|l|}{ Disease-specific information } \\
\hline Symptom management & 59 & 35 & 6 & 100 \\
\hline Prognosis & 23 & 18 & 1 & 42 \\
\hline Needs assessment & 11 & 15 & & 26 \\
\hline Epidemiology & 6 & & & 6 \\
\hline Psychosocial impact & 4 & & & 4 \\
\hline Stakeholder understanding of liver disease & 2 & 1 & & 3 \\
\hline New treatments & & 3 & & 3 \\
\hline \multicolumn{5}{|l|}{ Service model approaches } \\
\hline Optimal service structure & 14 & 5 & 2 & 21 \\
\hline Evaluating added value of palliative care & 8 & 5 & & 13 \\
\hline Barriers/improving access to SPC & 8 & & & 8 \\
\hline \multicolumn{5}{|l|}{ Education } \\
\hline How to improve management of liver patients & 3 & 3 & & 6 \\
\hline
\end{tabular}

SPC, specialist palliative care. 
patients for whom cure is no longer likely and to shift the emphasis of care from burdensome interventions to a more supportive care role. All groups recognise the need for collaborative working earlier in the illness so that discussions about the future can be raised at key points such as transplant assessments or exacerbations of symptoms. We identify symptom management, prognostication, and overall service structure as key areas for future research.

Our findings support key recommendations from a recent review of palliative care in end stage liver disease including better collaboration between liver health professionals and SPC and the identification of potential triggers for palliative care consultations. ${ }^{4}$ Our work also supports recommendations from the Lancet Commission report on the role of primary care in liver disease and the need for community hepatology posts for GPs to work more closely with liver professionals. $^{1}$

Lessons can be learned from research on the management of people with other advanced nonmalignant diseases. In the UK, in advanced heart failure $^{10}$ and advanced kidney disease ${ }^{11}$ shared care approaches have been developed involving multiprofessional collaboration between primary care and specialist services, and SPC input when required. The key elements are sensitive communication with patients and their families especially earlier in the illness, holistic assessment and care involving multiprofessional working across boundaries. While planning at a national level is not easy, on a local level, it would be important to improve education and coordination of service provision between liver specialists, GPs and SPC professionals.

We note the very low response rate to our survey, which currently limits the generalisability of our findings which require further validation. This low response rate may be due to limitations in our methods. Data protection issues prevented us from accessing mailing lists directly, which was the responsibility of the special interest groups, so the delivery of the mail-out was inconsistent. Email circulations may have been incomplete, and overlap in the membership of our targets groups (eg, BASL and the BSG liver section) may underestimate the response rate. However, our highest response rate of $24 \%$ achieved from SPC is still lower than the $33 \%{ }^{12}$ considered standard for on-line surveys. It is possible, but we cannot be certain that there is currently a reluctance to consider issues of EoL care among those working with people with cirrhosis. However, it is likely that the potential benefits of a palliative approach to care, either early and in parallel to more aggressive treatments, or in managing the terminal phase are not yet recognised.

Our response rates might have been higher had the research team been able to access directly the mailing list from each of the different organisations. This would have enabled the research team to check for duplicate participants and personalise the invitation letter before sending out to each health professional. We might also have been able to ensure that each non-responding professional received up to two follow-up emails to encourage their participation in the survey. Furthermore, if finances were available, we would have given participants on receipt of their response a non-monetary incentive such as Amazon gift cards to be used for educational purposes. Some of these initiatives have been identified in a Cochrane review as increasing response rates in electronic surveys. ${ }^{13}$

\section{CONCLUSION}

While there are plans to address the high mortality from liver disease, these initiatives will be slow to demonstrate any impact on mortality figures and many will continue to die with high symptom burden and little care planning at the EoL. The financial challenges faced by the NHS over the next years as outlined by the NHS England Chief Executive ${ }^{14}$ confirm that we cannot expect significant financial support to address the problem. We recommend research that takes account of economic costs and benefits, and improves our understanding of the needs of patients with cirrhosis and their families, enhances communication with those at risk of death, enables smoother links across care boundaries, and informs training for all professionals involved in treatments and care.

\section{Key messages}

What is already known about this subject?

- There is increasing number of deaths from liver disease in the UK.

- Most deaths from liver disease occur at a relatively young age and in patients with a higher degree of social deprivation.

- Access to palliative care for people with end stage liver disease (cirrhosis) has been limited and most deaths from cirrhosis occur in hospital.

\section{What this paper adds?}

- This study is the first UK national survey to specifically explore current attitudes and practices of different health professionals caring for patients with cirrhosis near the end of life, using on-line survey methodology.

- We identify symptom management, prognostication, and overall service structure as key areas for future research.

How might it impact on future clinical practice?

- Increase collaborative working between liver professionals and specialist palliative care to meet the needs of patients with end stage liver disease. 
Author affiliations

${ }^{1}$ Marie Curie Palliative Care Research Department, Division of Psychiatry, University College London, London, UK

${ }^{2}$ Department of Palliative Care, Royal Free London NHS Foundation Trust, London, UK

${ }^{3}$ Sheila Sherlock Liver Unit, Royal Free Hospital, London, UK

${ }^{4}$ University College London Institute of Liver and Digestive Health, UCL Royal Free Campus, London, UK

Acknowledgements The authors thank the following for their contribution to this study: Mr Andrew Langford (British Liver Trust) and Professor Paddy Stone (UCL) for advice and contribution; Mr Howard Ellison (British Society for Gastroenterology), Ms Paula Banks (British Association for the Study of the Liver), Ms Kirsty Moulsley (Primary Care Society for Gastroenterology), Ms Becki Munroe (Association of Palliative Medicine) and Ms Hazel Caroll (British Liver Nurses Forum) for organising the survey mail-out to members of their respective organisations. The authors acknowledge Marie Curie for funding the Department responsible for developing and conducting this study.

Contributors JL, VV and SD were responsible for the conception and design of this work, acquisition, analysis and interpretation of data, drafting the work and giving final approval. JB was responsible for the design of the work, data analysis and interpretation, drafting the work and giving final approval. LG, KH, AM, DT and LJ were responsible for the design of this work, revising it critically for important content, and giving final approval.

Competing interests None declared.

Ethics approval UCL Ethics committee (3552/002).

Provenance and peer review Not commissioned; externally peer reviewed.

Data sharing statement Extra data is available by emailing Dr Joe Low at joseph.low@ucl.ac.uk.

\section{REFERENCES}

1 Williams R, Aspinall R, Bellis M, et al. Addressing liver disease in the UK: a blueprint for attaining excellence in health care and reducing premature mortality from lifestyle issues of excess consumption of alcohol, obesity, and viral hepatitis. Lancet 2014;384:1953-97.

2 Effong K, Osinowo A, Pring A. Verne J Deaths From Liver Disease: Implications for End of Life Care in England.
National End of life Care Intelligence Network, Leicester. 2012 http://tinyurl.com/bogzgsu (accessed 13 Feb 2015).

3 National Health Service Right Care. Liver disease: the NHS atlas of variation in healthcare for people with liver disease. 2013. http://www.rightcare.nhs.uk/index.php/atlas/liver-diseasenhs-atlas-of-variationin-healthcare-for-people-with-liver-disease (accessed 22 Jan 2015).

4 Potosek J, Curry M, Buss M, et al. Integration of palliative care in end-stage liver disease and liver transplantation. J Palliat Med 2014;17:1271-7.

5 Roth K, Lynn J, Zhong Z, et al. (2000). Dying with end stage liver disease with cirrhosis: insights from SUPPORT. Study to Understand Prognoses and Preferences for Outcomes and Risks of Treatment. J Am Geriatr Soc 2000;48(5 Suppl): S122-30.

6 Sanchez W, Talwalker JA. Palliative care for patients with end-stage liver disease ineligible for liver transplantation. Gastroenterol Clin North Am 2006;35:201-19.

7 Hansen L, Sasaki A, Zucker B. End-stage liver disease: challenges and practice implications. Nurs Clin North Am 2010;45:411-26.

8 National Confidential Enquiry into Patient Outcome and Death (NCEPOD). Alcohol-related liver disease: measuring the units. 2013. http://www.ncepod.org.uk/2013arld.htm (accessed 13 Feb 2015).

9 StataCorp. 2013. Stata Statistical Software: Release 13. College Station, TX: StataCorp LP.

10 Scottish Partnership for Palliative Care. Living and dying with advanced heart failure: a palliative care approach. Edinburgh. 2008. http://www.palliativecarescotland.org.uk/ content/publications/HF-final-document.pdf (accessed 20 Feb 2015).

11 NHS Kidney Care. (2009b). End of life care in advanced kidney disease: a framework for implementation. http://www. ncpc.org.uk/sites/default/files/EndOfLifeCareInAdvanced KidneyDisease.pdf (accessed 13 Feb 2015).

12 Nulty DD. The adequacy of response rates to online and paper surveys: what can be done? Assess Eval Higher Educ 2008;33:301-14.

13 Edwards PJ, Roberts I, Clarke MJ, et al. Methods to increase response to postal and electronic questionnaires. Cochrane Database Syst Rev 2009, (3):MR000008. DOI:10.1002/ 14651858.MR000008.pub4.

14 NHS England. Five year forward view 2014. http://www. england.nhs.uk/wp-content/uploads/2014/10/5yfv-web.pdf (assessed 6 Feb 2015). 